\section{Electronic Spectra of Antimony Monobromide Part II *}

M. N. Avasthi, A. K. Sharma, and (Miss) A. R. Sud

(Z. Naturforsch. 30 a, 695-696 [1975] ; received March 8,1975 )

A new group of bands, designated as system A, of antimony monobromide, lying in the region $5340-4905 \AA$, has been photographed and completely analysed. Two characteristic continua with maxima at $5320 \AA$ and $6500 \AA$ have been observed. The electronic states with the recorded band systems have been tentatively assigned.

In an earlier paper (1971) the senior author had analysed the band structures of the two band systems lying in the regions $3340-3050 \AA$ and $3020-$ $2950 \AA$. These were designated as systems $B_{1}$ and $B_{2}$ respectively.

Another system, extending from $5340-4905 \AA$, was also mentioned. Heretofore, it will be designated as system $\mathrm{A}$. The general characteristics of this system are the same as those of systems $B_{1}$ and $B_{2}$. The vibrational analysis of this new system and a general consideration of the electronic structure of the molecule are the subject matter of this paper.

$$
\text { System } A(5340-4905 A)
$$

Using the same experimental set up and conditions as described earlier, the band system under discussion was photographed with a Fuess spectrograph. In this region a large number of atomic lines have been recorded, indicating a good deal of dissociation of the molecule into its constituent elements. Two continua, one situated just at the long wavelength end of the observed discrete band system with a maximum at about $5320 \AA$, and the other near $6500 \AA$, have been observed. An enlargement of the spectrum is given in Figure 1.
The wavenumbers in vacuum of the band heads, their visually estimated relative intensities, vibrational analysis along with $\nu$ obs. and $\mathrm{O}-\mathrm{C}$ values are given in Table 1 . All the bands are degraded towards the longer wavelength side.

Table 1. Band head data and vibrational anlaysis of the system A $(5340-4905 \AA)$.

\begin{tabular}{lllr}
\hline $\begin{array}{l}\text { Wave number in } \\
\text { vacuum }\left(\mathrm{cm}^{-1}\right)\end{array}$ & $\begin{array}{l}\text { Relative } \\
\text { intensity }\end{array}$ & $v^{\prime}, v^{\prime \prime}$ & $\begin{array}{l}\text { O-C values } \\
\left(\mathrm{cm}^{-1}\right)\end{array}$ \\
\hline 18732 & 8 & 2,6 & 10 \\
18966 & 5 & 2,5 & 5 \\
18984 & 8 & 1,4 & -2 \\
19200 & 8 & 2,4 & -1 \\
19230 & 7 & 1,3 & 4 \\
19253 & 3 & 0,2 & 1 \\
19375 & 4 & 4,5 & -12 \\
19445 & 3 & 2,3 & 4 \\
19470 & 8 & 1,2 & 2 \\
19494 & 6 & 0,1 & 0 \\
19612 & 3 & 4,4 & -15 \\
19706 & 8 & 1,1 & -3 \\
19736 & 7 & 0,0 & 0 \\
19771 & 1 & - & -8 \\
19831 & 2 & 5,4 & 5 \\
19900 & 3 & 3,2 & 0 \\
19951 & 2 & 1,0 & 3 \\
20140 & 2 & 3,1 & 0 \\
20166 & 2 & 2,0 & 1 \\
20380 & 9 & 3,0 & \\
\hline
\end{tabular}

A faint line lying on a continuum sometimes gives the impression of a band. Whenever such a suspicion arose, the authors have preferred not to mention it in the above table. The total number of such bands is four.

The Franck-Condon parabola representing the relative intensity distribution in the band system is similar to that obtained in the system $B_{1}$. The values

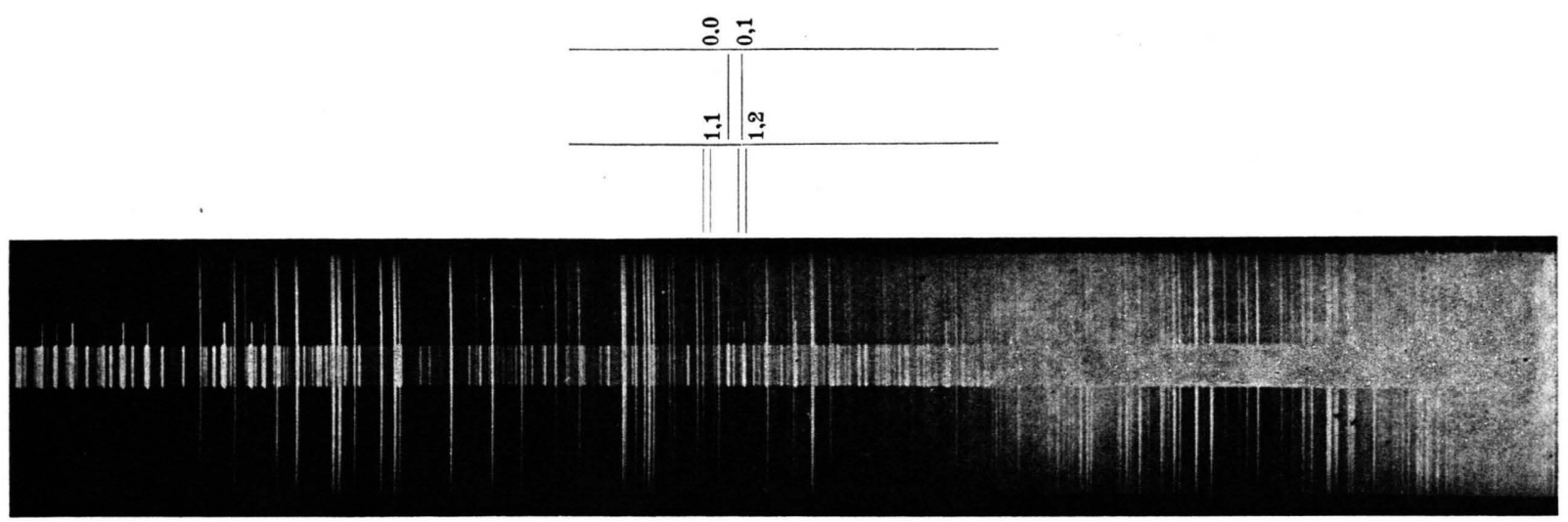

Fig. 1. Emission spectrum of $\mathrm{SbBr}$ molecule.

* This work was done at Banaras Hindu University, Varanasi (India) and subsequently repeated at the University of Jodhpur, Jodhpur (India). 
of $\omega_{\mathrm{e}}^{\prime}$ and $\omega_{\mathrm{e}}{ }^{\prime \prime}$ for the states involved conform with the nature of the $\mathrm{F}-\mathrm{C}$ parabola.

The $\Delta G^{\prime \prime}(v+1 / 2)$ values clearly show that the lower state of this system can be identified with the lower state of the systems $B_{1}$ and $B_{2}$. This fact was taken into account while assigning $v^{\prime \prime}$ values to different bands of the system. The value of $\omega_{\mathrm{e}}{ }^{\prime \prime} \mathrm{ob}$ tained tallies very well with that obtained for the systems $B_{1}$ and $B_{2}$.

All the bands of the system A can fairly well be represented by the following equation:

$$
\begin{aligned}
v=19736+\left(215.45 v^{\prime}\right. & \left.-0.35 v^{\prime 2}\right) \\
& -\left(242.6 v^{\prime \prime}-0.33 v^{\prime \prime 2}\right) .
\end{aligned}
$$

The $\omega_{\mathrm{e}}$ values are correct to $\pm 3 \mathrm{~cm}^{-1}$, while the $\omega_{\mathrm{e}} \chi_{\mathrm{e}}$ values are correct to the first decimal place only.

On the basis of a comprehensive discussion (Ph. D. Thesis (1964) of the senior author, submitted at Banares Hindu University, Varanasi, India) the ground state of the molecule was suggested to be ${ }^{3} \Sigma^{-1}$ and the two excited states to be ${ }^{3} \Sigma^{-1}$ and

1 W. F. C. Ferguson and I. Hudes, Phys. Rev. 57, 705 [1940].

2 G. Herzberg, Molecular Spectra and Molecular Structure Spectra of Diatomic Molecules, D. Van Nostrand Company, Inc., New York 1950.
${ }^{3} \Pi$. The bands system was attributed to the following transitions.

System A

System $B\left(B_{1}\right.$ and $\left.B_{2}\right) \quad{ }^{3} \Pi \rightarrow{ }^{3} \Sigma^{-1}$ (ground state).

It is well known that a correct species of electronic states involved in the different transitions can only be decided by rotational analysis. Since the molecule is heavy, the bands are slightly diffuse, and $\mathrm{SbBr}_{3}$ is deliquescent, the rotational analysis is not practically possible. Hence the assignments may only be treated as tentative.

\section{Acknowledgement}

The authors are thankful to Prof. N. L. Singh and Prof. D. K. Rai of Physics Department, Banares Hindu University, Varanasi (India) for valuable discussions and suggestions and to Prof. A. N. Nigam of Jodhpur University for giving facilities and continuous encouragement. One of us, Miss A. R. Sud is grateful to U.G.C. (India) for the award of Junior Research Fellowship.

3 M. N. Avasthi, Z. Naturforsch. 26 a, 250 [1971].

4 G. R. M. I. T. Harrison, Wavelength Tables, John Wiley and Sons, New York 1939. 